\title{
Radiosurgery for multiple brain metastases: two cases reports
}

\begin{abstract}
Brain metastases are the most common intracranial tumors in the adult population and historically treated with whole brain radiation therapy (WBRT). However, as medical oncology advances improve life expectancy, stereotactic radiosurgery (SRS) has replaced WBRT as the standard for limited brain metastases, 1-3 nevertheless has been less published in the case of multiple brain metastases. In these cases reports, we detail two patients at our institution that received SRS for multiple brain metastases and demonstrated acceptable tolerance and response. SRS is emerging as an acceptable alternative in multiple brain metastases and the decision to indicate it should be made with consideration of overall prognosis for each patient.
\end{abstract}

Keywords: brain metastases, stereotactic radiosurgery, LINAC, frameless
Volume 8 Issue 4 - 2018

\author{
Caussa L,' Davalos D,' Perez Conci O,' \\ Franco D,' Aon Egle,' Garrigo E,' Fernandez \\ D, ' Calle Cueva R, ${ }^{4}$ Gonzalez D, ${ }^{2}$ Herrera E, ${ }^{3}$ \\ Diaz Vasquez M,' Ferraris $G^{\prime}$ \\ 'Centro Médico Deán Funes, Córdoba, Argentina \\ ${ }^{2}$ Hospital Ferreyra, Córdoba, Argentina \\ ${ }^{3}$ Sanatorio Allende, Córdoba, Argentina \\ ${ }^{4}$ Instituto Peruano de Oncología y Radioterapia, Perú
}

Correspondence: Caussa Lucas, Dean Funes 2869, Cordoba, Argentina, Tel 005493517670253 ,

Email lucas.caussa@dfunes.com.ar

Received: March 29, 2018| Published: August 08, 2018
Abbreviations: WBRT, whole brain radiation therapy; SRS, stereotactic radiosurgery; OS, overall survival; LINAC, linear accelerator; IMRT, intensity radiotherapy technique

\section{Introduction}

Brain metastases are diagnosed in to $20-40 \%$ of patients diagnosed with cancer and whole brain radiation therapy (WBRT) has been the standard treatment, but associated with deleterious effects on neurocognitive function and quality of life. ${ }^{1,2}$ Stereotactic radiosurgery (SRS) has become as a means to treat this selected patients groups.

Actually, WBRT remains the standard approach for treatment of patients with multiple brain metastases. However, new nonrandomized studies evaluating SRS alone in patients with four or more brain metastases have shown comparable overall survival (OS) to WBRT. Therefore, SRS to multiple lesions in a single session has evolved to become a new alternative to WBRT with good favorable overall prognosis..$^{4-7}$ In this article, we report the outcomes of SRS for multiple brain metastases in select cases.

\section{Case presentations}

\section{Patient \# I}

A 63-year-old woman with a history of breast cancer of 5years of evolution with bones metastasis controlled with systemic treatment and 2years later presented cerebral metastases treated with WBRT sparing both hippocampus (20Gy in 5 fractions). One year at the end she has new brain progression without neurological symptoms and controlled systemic disease. We evaluated the case and in view of the good general condition we indicated re-irradiation with radiosurgery to all visible lesions: 83 (eighty-three) in total (Figure1). The total volume of disease was $34 \mathrm{cc}$ and the dose prescription of SRS 27Gy in 3 fractions. For the treatment, we used a dedicated linear accelerator (LINAC) Trilogy Varian, frame less immobilization and RapidArc modulated intensity radiotherapy technique (IMRT) with an treatment isocenter. After 6months of follow-up, there was no neurocognitive impairment or alteration of quality of life, without systemic progression and in the brain resonance complete response was seen in $90 \%$ of the lesions with tumor control of all the metastases (Figure2).

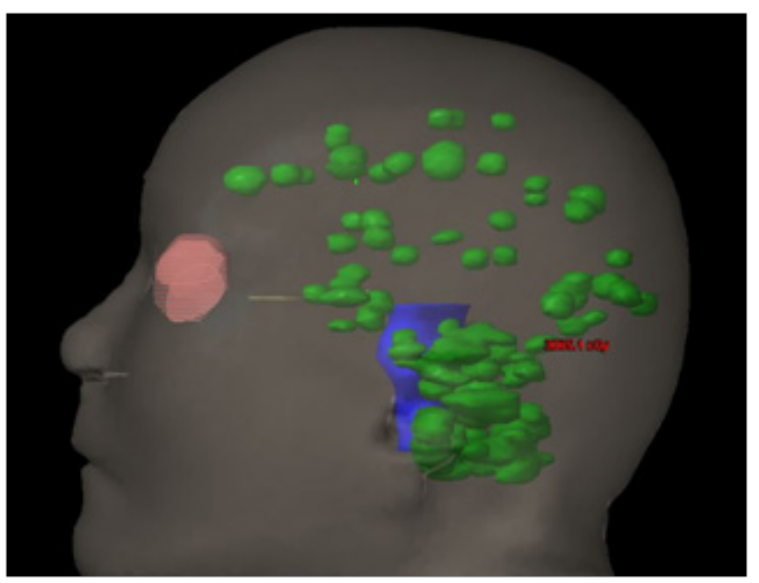

Figure I 83 metastases, 3D reconstruction.

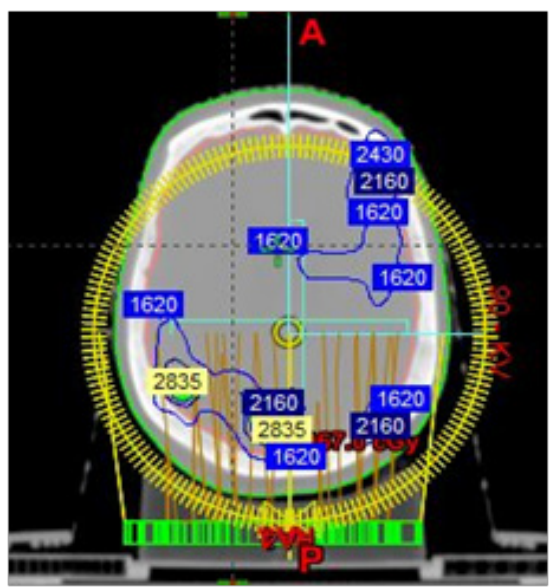

Figure 2 RapidArc IMRT technique. 


\section{Patient \#2}

30-year-old man, with lung cancer treated with chemotherapy and complete response to his primary tumor. In extension images studies at 6months he presents multiple brain metastases without evidence of progression in another site. We propose SRS to all visible lesions: 24 (twenty-four). The total volume of disease was $15 \mathrm{cc}$ and the dose prescription of SRS was 27Gy in 3 fractions (Figure 3) (Figure 4). The treatment technique for this case was the same as that used for the previous patient. After lyear of follow-up, there was no neurocognitive impairment without systemic progression and in the brain resonance complete response was seen in $100 \%$ of the lesions.

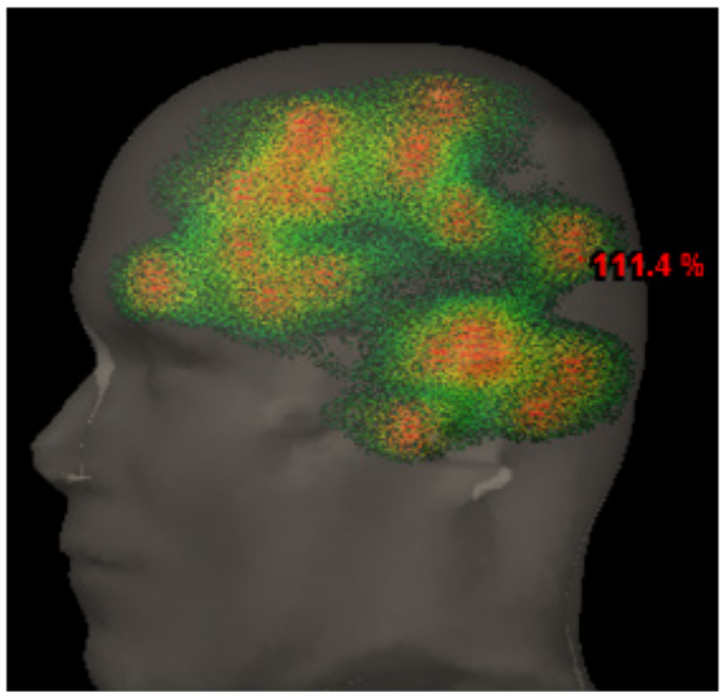

Figure 324 metastases, dose distributions.

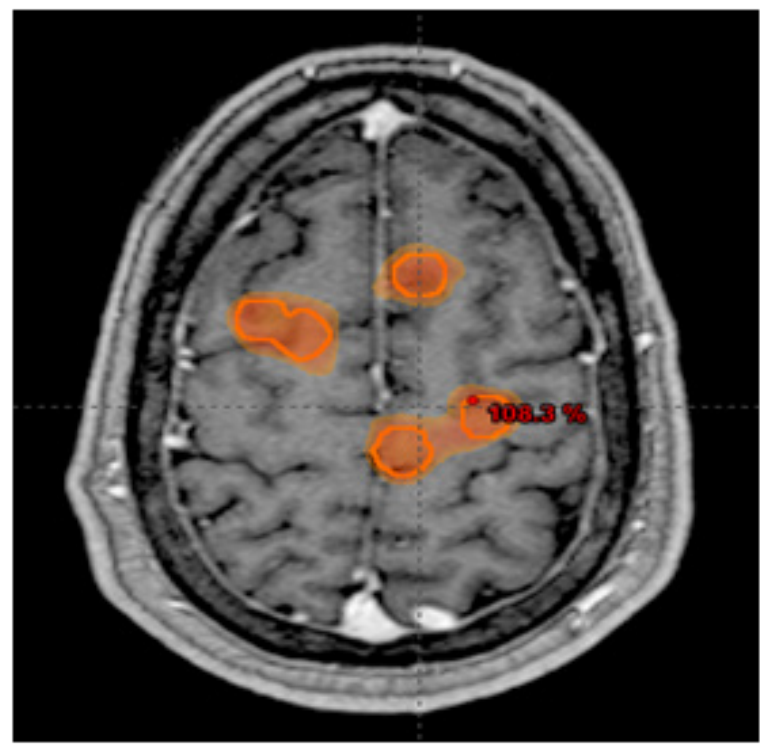

Figure 4 RapidArc IMRT technique.

\section{Discussion}

Technologic advances in treatment delivery and treatment planning allowing for shorter treatment facilite the use of SRS alone as the primary treatment for patients with more than four lesions with acceptable tolerance and local control. ${ }^{8,9}$ Factors such as a cumulative tumor volume, Karnofsky Performance Status, and Age, were better potential predictors of long-term survival on multivariable analysis rather than number of lesions alone. This can be used to identify the patients who would benefit the most from SRS. Nevertheless, patients who have regional failure after WBRT can undergo effective salvage SRS with low morbidity and may have similar outcomes to those who experience first brain metastases with similar performance status. ${ }^{6,10-15}$ Local treatment should be evaluated for patients with multiple brain metastases and good performance status for whom salvages SRS is technically feasible.

\section{Conclusions}

These are two cases reports of selected patients with multiple brain metastases that have exceptional outcomes after SRS. Several factors such as performance status, tumor volume and systemic disease are important for treatment election.

\section{Acknowledgements}

None.

\section{Conflict of interest}

The author declares no conflict of interest.

\section{References}

1. Patchell RA, Tibbs PA, Walsh JW, et al. A randomized trial of surgery in the treatment of single metastases to the brain. NEngl J Med. 1990;322(8):494500 .

2. Patchell RA, Tibbs PA, Regine WF, et al. Postoperative radiotherapy in the treatment of single metastases to the brain: a randomized trial. JAMA. 1998;280(17):1485-1489.

3. Linskey ME, Andrews DW, Asher AL, et al. The role of stereotactic radiosurgery in the management of patients with newly diagnosed brain metastases: a systematic review and evidence-based clinical practice guideline. J Neurooncol. 2010;96(1):45-68.

4. Yoshio K, Mitsuhashi T, Wakita A, et al. Plan quality comparison between 4-arc and 6-arc noncoplanar volumetric modulated arc stereotactic radiotherapy for the treatment of multiple brain metastases. Med Dosim. 2018;pii:S0958-3947(17)30134-6.

5. Ohira S, Ueda Y, Akino Y, et al. HyperArc VMAT planning for single and multiple brain metastases stereotactic radiosurgery: a new treatment planning approach. Radiat Oncol. 2018;13(1):13.

6. Smith DR, Wu CC, Wang TJC. Stereotactic radiosurgery for treatment of multiple brain metastases: Remembering to spare the hippocampus?: In Regard to Zhang et al. Pract Radiat Oncol. 2017;7(6):446-447.

7. Sahgal A, Ruschin M, Ma L, et al. Stereotactic radiosurgery alone for multiple brain metastases? A review of clinical and technical issues. Neuro Oncol.2017;19(suppl_2):ii2-ii15.

8. Yamamoto M, Serizawa T, Higuchi $\mathrm{Y}$, et al. A multi-institutional prospective observational study of stereotactic radiosurgery for patients with multiple brain metastases (JLGK0901 study update): Irradiationrelated complications and long-term maintenance of mini-mental state examination scores. Int J Radiat Oncol Biol Phys. 2017;99(1):31-40.

9. Yamamoto M, Serizawa T, Shuto T, et al. Stereotactic radiosurgery for patients with multiple brain metastases (JLGK0901): a multi-institutional prospective observational study. Lancet Oncol. 2014;15(4):387-395. 
10. Sperduto W, King DM, Watanabe Y, et al. case report of extended survival and quality of life in a melanoma patient with multiple brain metastases and review of literature. Cureus. 2017;9(12):e1947.

11. Robin TP, Camidge DR, Stuhr K, et al. excellent outcomes with radiosurgery for multiple brain metastases in ALK and EGFR driven non-small cell lung cancer. J Thorac Oncol. 2018;13(5):715-720.

12. Pham A, Lee B, Chang EL. Stereotactic radiosurgery for multiple brain metastases: two cases of preserved quality of life. Cureus. 2017;9(12):e1995.
13. Miller JA, Kotecha R, Barnett GH, et al. Quality of life following stereotactic radiosurgery for single and multiple brain metastases. Neurosurgery. 2017;81(1):147-155.

14. Logie N, Jimenez RB, Pulenzas N, et al. Estimating prognosis at the time of repeat whole brain radiation therapy for multiple brain metastases: The reirradiation score. Adv Radiat Oncol. 2017;2(3):381-390.

15. Kalash R, Pifer PM, Beriwal S, et al. Exceptional eight-year response to stereotactic radiosurgery monotherapy for multiple brain metastases. Cureus. 2017;9(12): e2001. 